DRAFT VERSION JUNE 11,2018

Preprint typeset using LTEX style emulateapj v. 6/22/04

\title{
CALIBRATION OF THE OPTICAL MASS PROXY FOR CLUSTERS OF GALAXIES AND AN UPDATE OF THE WHL12 CLUSTER CATALOG
}

\author{
Z. L. WEN, J. L. HAN \\ National Astronomical Observatories, Chinese Academy of Sciences, 20A Datun Road, Chaoyang District, Beijing 100012, China; zhonglue@nao.cas.cn. \\ Draft version June 11, 2018
}

\begin{abstract}
Accurately determining the mass of galaxy clusters is fundamental for many studies on cosmology and galaxy evolution. We collect and rescale the cluster masses of 1191 clusters of $0.05<z<0.75$ estimated by $\mathrm{X}$-ray or Sunyaev-Zeldovich measurements and use them to calibrate the optical mass proxy. The total $r$-band luminosity (in units of $L^{*}$ ) of these clusters are obtained by using spectroscopic and photometric data of the Sloan Digital Sky Survey (SDSS). We find that the correlation between the cluster mass $M_{500}$ and total $r$-band luminosity $L_{500}$ significantly evolves with redshift. After correcting for the evolution, we define a new cluster richness $R_{L *, 500}=L_{500} E(z)^{1.40}$ as the optical mass proxy. By using this newly defined richness and the recently released SDSS DR12 spectroscopic data, we update the WHL12 cluster catalog and identify 25,419 new rich clusters at high redshift. In the SDSS spectroscopic survey region, about $89 \%$ of galaxy clusters have spectroscopic redshifts. The mass can be estimated with a scatter of 0.17 dex for the clusters in the updated catalog.
\end{abstract}

Subject headings: galaxies: clusters: general — galaxies: distances and redshifts

\section{INTRODUCTION}

Clusters of galaxies are the most massive gravitationally bound systems in the universe. Statistics of cluster properties provides very powerful constraints on cosmology and galaxy evolution (see Allen et al. 2011; Wetzel et al. 2012). The cluster mass is a fundamental parameter for galaxy clusters and is related to observational features in the X-ray, optical, millimeter and radio bands (e.g., Voit 2005; Brunetti et al. 2009). Galaxies, intracluster hot gas and dark matter contribute about $5 \%, 15 \%$, and $80 \%$ of the total cluster mass, respectively. Determining the cluster mass is the basis for many studies. For example, the mass function for a complete sample of galaxy clusters can be used to constrain cosmological parameters (e.g., Reiprich \& Böhringer 2002; Vikhlinin et al. 2009b; Wen et al. 2010).

Statistics for weak gravitational lensing features is the most direct method to determine the total cluster mass including contributions from member galaxies, hot intracluster gas and dark matter. However, this method requires high-quality image data and also redshift information for faint background galaxies and thus only a few massive clusters have their masses so determined (e.g., Dahle 2006; Bardeau et al. 2007; Okabe et al. 2010; von der Linden et al. 2014b). Based on the assumption that the hot intracluster gas is in hydrostatic equilibrium, the cluster mass can be estimated by using the X-ray surface brightness and temperature distributions (e.g., Finoguenov et al. 2001; Reiprich \& Böhringer 2002; Kravtsov et al. 2006; Vikhlinin et al. 2006). A few thousands of galaxy clusters have their masses estimated from the X-ray observables (Vikhlinin et al. 2009a; Mantz et al. 2010; Piffaretti et al. 2011; Takey et al. 2013). In the millimeter band, the thermal Sunyaev-Zeldovich (SZ) effect provides robust mass estimates for more than one thousand massive clusters (Hasselfield et al. 2013; Reichardt et al. 2013; Planck Collaboration et al. 2014), which is independent of cluster dynamical state and redshift (Carlstrom et al. 2002).

The velocity dispersion of member galaxies is certainly a good measure of cluster mass (Girardi et al. 1998), which can be derived from optical spectroscopic data and have been ob- tained for a limited number of galaxy clusters. However, the spectroscopic observations are usually incomplete for cluster member galaxies, and cluster substructures often induce a bias on the estimated mass (Bird 1995; Sifón et al. 2013). On the other hand, photometric data can be used to identify galaxy clusters (e.g., Koester et al. 2007; Wen et al. 2009; Hao et al. 2010; Szabo et al. 2011; Wen et al. 2012; Oguri 2014; Rykoff et al. 2014). Cluster richness, defined as the total number of member galaxies or their total luminosity, can be used as an optical mass proxy (Popesso et al. 2005, 2007). The challenge is to accurately determine the membership of cluster galaxies by eliminating the contamination by field galaxies. Based on multicolor survey data, the member galaxies can be discriminated by using galaxy colors (e.g., Gladders \& Yee 2000; Koester et al. 2007; Hao et al. 2010) or photometric redshifts (e.g., Wen et al. 2009; Szabo et al. 2011). The cluster richness derived from optical photometric data in general is poorly correlated with cluster mass. However, if the correlation is improved, the cluster richness can be used to estimate cluster mass for a very large sample of clusters even up to high redshifts.

The Sloan Digital Sky Survey (SDSS; York et al. 2000) offers an unprecedented photometric data in five broad bands ( $u, g, r, i$, and $z$ ) covering $14,000 \mathrm{deg}^{2}$ with the exceptional follow-up spectroscopic observations. The photometric data reach a limit of $r=22.2$ (Stoughton et al. 2002), with the star-galaxy separation reliable to a limit of $r=$ 21.5 (Lupton et al. 2001). The spectroscopic survey observes galaxies with a Petrosian magnitude of $r<17.77$ for the main galaxy sample (Strauss et al. 2002) and a model magnitude $r<19.9$ for the Luminous Red Galaxy sample (Eisenstein et al. 2001). These galaxies cover a footprint of $10,400 \mathrm{deg}^{2}$ in the range of $-11^{\circ} \leq$ Decl. $\leq 69^{\circ}$. The latest Data Release 12 (DR12, Alam et al. 2015) includes the photometric data for about 200 million galaxies and the spectroscopic data for about 2.32 million galaxies.

The large samples of galaxy clusters or groups have been identified by using the SDSS spectroscopic data (e.g., Merchán \& Zandivarez 2005; Berlind et al. 2006; 
Tempel et al. 2014) and the photometric data (e.g., Koester et al. 2007; Wen et al. 2009; Hao et al. 2010; Szabo et al. 2011; Wen et al. 2012; Oguri 2014; Rykoff et al. 2014). Generally, the richness in these cluster catalogs is defined as the total number or the total luminosity of member galaxies, which can be regarded as a coarse mass proxy for clusters up to a redshift of $z \sim 0.4$. At higher redshifts, the discrimination of member galaxies are limited to only very luminous galaxies, and hence the richness is systematically underestimated, as discussed in Wen et al. (2012). We noticed that without a proper calibration, such a cluster richness could be redshift-dependent even at low redshift (e.g., Rozo et al. 2009; Wen et al. 2010).

Many recent samples of galaxy clusters have their masses determined by X-ray or SZ measurements up to redshift of $z \sim 0.75$ (e.g., Piffaretti et al. 2011; Planck Collaboration et al. 2014). These provide an opportunity to calibrate the richness over a large redshift range so that the calibrated richness may be used as a mass proxy for a very large sample of galaxy clusters. In Section 2, we collect the cluster mass in the literature estimated by the X-ray or SZ observations, and for these clusters in the SDSS survey region, we calculate their total $r$-band luminosity and define a mass proxy that is independent of redshift. On the other hand, the SDSS Baryon Oscillation Spectroscopic Survey (Dawson et al. 2013) offers spectroscopic redshifts for most of the galaxy clusters in the catalog of Wen et al. (2012, i.e., the WHL12 cluster catalog), so that in Section 3 we update the richness and redshift information for the WHL12 clusters, and find new complementary clusters at high redshift.

Throughout this paper, we assume a $\Lambda \mathrm{CDM}$ cosmology, taking $H_{0}=100 h \mathrm{~km} \mathrm{~s}^{-1} \mathrm{Mpc}^{-1}$, with $h=0.7, \Omega_{m}=0.3$ and $\Omega_{\Lambda}=0.7$.

\section{CALIBRATING THE RICHNESS FOR AN OPTICAL MASS PROXY}

We collect the estimated masses of many large samples of galaxy clusters, and then scale them to each other by using common clusters. The scaled masses of the composite sample of galaxy clusters are used to calibrate the optical richness of galaxy clusters in a large range of redshifts so that any redshift dependence can be eliminated.

\subsection{The mass estimates for galaxy clusters and their rescaling between different samples}

Many samples of galaxy clusters have their mass estimated by using the X-ray or SZ measurements (e.g. Kravtsov et al. 2006; Zhang et al. 2008; Pratt et al. 2009; Zhao et al. 2013). To make the largest composite sample with unified scaling on mass estimates, however, we collect only large samples of clusters from the literature, with each having at least 10 overlapping common clusters with the other samples so that the estimated masses of these common clusters can be compared and scaled. For this purpose, we take six large samples from the literature: the X-ray cluster samples by Vikhlinin et al. (2009a, hereafter V09), Mantz et al. (2010, hereafter M10), Piffaretti et al. (2011, hereafter P11) and Takey et al. (2013, 2014, hereafter T14), and the SZ cluster samples from the Atacama Cosmology Telescope (ACT) survey by Hasselfield et al. (2013, hereafter H13) and the Planck survey by the Planck Collaboration et al. (2015, hereafter P15). We extract the cluster mass $M_{500}$ from these references, which is the mass within a radius of $r_{500}$ within which the mean density of a cluster is 500 times of the critical den- sity of the universe, $\rho_{c} \equiv 3 H^{2}(z) / 8 \pi G$, i.e.

$$
M_{500}=\frac{4 \pi}{3} r_{500}^{3} \times 500 \rho_{c} .
$$

In the following we briefly introduce the six samples:

V09: Vikhlinin et al. (2009a) estimated the total masses for 85 clusters at $z \approx 0.05$ and $z \approx 0.5$ by using highquality Chandra observations. The cluster mass $M_{500}$ was derived by using three $\mathrm{X}$-ray proxies: average temperature $T_{X}$, gas mass $M_{\text {gas }}$, and $Y_{X}=T_{X} \times M_{\text {gas }}$. Vikhlinin et al. (2009a) calibrated their scaling relations with cluster mass (Kravtsov et al. 2006) by using a sample of well-observed relaxed clusters at low redshifts. The mass estimates derived using the three proxies are well matched with very small scatters. We adopt the cluster mass $M_{500}$ they derived from $M_{\text {gas }}$ and the gas mass fraction, which depends weakly on cluster mass.

M10: Mantz et al. (2010) used high-quality Chandra observations to estimate the gas mass $M_{\text {gas }}$ for 130 massive clusters that have $0.1-2.4 \mathrm{keV}$ luminosity larger than $2.5 \times 10^{44} \mathrm{erg} \mathrm{s}^{-1}$ in the redshift range of $0.05<z<0.88$. Different from V09, they derived the total mass $M_{500}$ assuming a constant value of gas mass fraction.

P11: Piffaretti et al. (2011) compiled 1743 clusters in the redshift range of $z<1.2$ from the ROSAT All Sky Surveybased data, including NORAS (Böhringer et al. 2000), REFLEX (Böhringer et al. 2004), BCS (Ebeling et al. 1998), SGP (Cruddace et al. 2002), NEP (Henry et al.|2006), MACS (Ebeling et al. 2001) and CIZA (Ebeling et al. 2002), and serendipitous searches in the ROSAT observations including 160SD (Mullis et al. 2003), 400SD (Burenin et al. 2007), SHARC (Romer et al. 2000), WARPS (Horner et al. 2008) and EMSS (Gioia et al. 1990). All clusters have their X-ray luminosity, $L_{X, 500}$, measured within $r_{500}$. The cluster masses were estimated from $L_{X, 500}$ by using the scaling relation between mass and X-ray luminosity obtained by Arnaud et al. (2010) and Pratt et al. (2009), with a typical uncertainty of $15 \%$.

T14: Takey et al. (2013) presented a sample of 530 X-ray clusters selected from the XMM-Newton serendipitous source catalog in the SDSS sky region, of which 310 clusters have spectroscopic redshifts and are in the redshift range of $0.03<$ $z<0.70$. Takey et al. (2014) updated the photometric redshifts of the clusters by using the SDSS DR10 spectroscopic data. Cluster masses are estimated by using the X-ray luminosity $L_{X, 500}$ and the scaling relations given by Pratt et al. (2009).

H13: Hasselfield et al. (2013) presented a catalog of 68 clusters over $0.15<z<0.8$ detected via SZ effect at $148 \mathrm{GHz}$ in the ACT survey. The authors provided cluster mass estimates for the 68 clusters near the celestial equator together with 23 clusters in the southern survey. Four different mass estimates were given in the paper based on the Compton parameter calibrated to the Universal Pressure Profile model (Arnaud et al. 2010), the Bode et al. (2012) model, the Nonthermal model (Trac et al. 2011) and the dynamical masses of Sifón et al. (2013), respectively. These four mass estimates are well correlated with different normalization. Here, we adopt the mass estimates calibrated to dynamical masses of Sifón et al. (2013). 

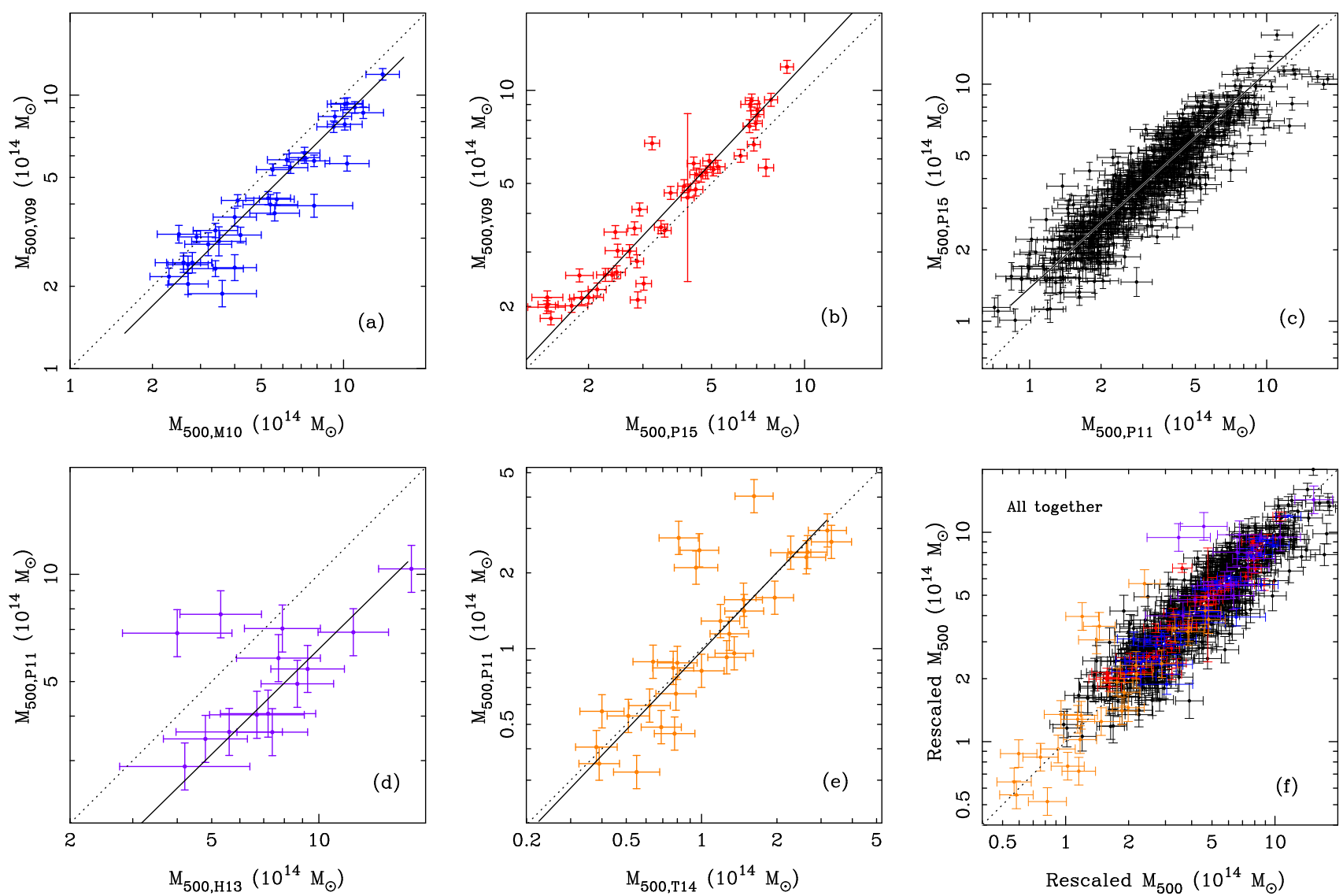

FIG. 1.-Comparison of the estimated masses for the overlapping common clusters in different samples from the literature. The solid line is the best fit, and the dotted line indicates the equivalence. All data in panels (a) - (e) are finally rescaled to V09 via the best fit(s), and summarized in panel (f), where the mass values in the $\mathrm{X}$-axis are rescaled from the X-values of panels (a) - (e) and the mass values in the Y-axis are rescaled from the Y-values of panels (a) - (e). Because the intrinsic uncertainty of mass-observable relation is larger than 5\% (Vikhlinin et al. 2009a), we here set the minimum uncertainty of 5\% for cluster mass even though it could be formally small in the literature.

P15: The all-sky Planck catalog of clusters is the largest SZ-selected catalog to date, containing 1653 clusters with redshifts up to $z \sim 1$ in the second catalog (Planck Collaboration et al. 2015). Among these, 1203 clusters have counterparts in previous X-ray, optical, SZ, and infrared data and 1094 clusters have redshifts. The masses of the 1094 clusters with redshift information were derived by using the Compton parameter (Planck Collaboration et al. 2014).

The masses of galaxy clusters in these samples are compiled to form a very large composite sample. However, we notice that cluster masses in these six samples were estimated by using different mass proxies with different scaling relations, and even cluster masses derived from different X-ray proxies in the same sample are not consistent as well. Though both V09 and M10 derived cluster masses via the gas mass $M_{\text {gas }}$, normalization of the fraction of gas mass and dependence on cluster mass are different in the two papers. Different cluster masses are obtained in $\mathrm{H} 13$ if they are normalized to different models. Therefore, to get the uniformed scale for estimated masses of galaxy clusters in a composite sample, the mass estimates in different samples by different authors must be rescaled to each other for consistency.

In general, the mass estimates derived by the proxies of $M_{\text {gas }}$ and $Y_{X}$ are more reliable than those from the $\mathrm{X}$ ray luminosity (Kravtsov et al. 2006). For the SZ clusters, we find that the mass estimates depend on model for the ACT clusters (Hasselfield et al. 2013); the cluster masses from the Planck survey are probably systematically underestimated (Planck Collaboration et al. 2014; von der Linden et al. 2014a). In this work, we rescale cluster masses to the standard obtained by Vikhlinin et al. (2009a) because their mass estimates are based on very high-quality X-ray data and the mass proxy shows only a small scatter after careful calibration using the known masses of low redshift relaxed clusters.

The easy approach to rescale the cluster masses to the standard of Vikhlinin et al. (2009a) is to compare the mass values of common clusters that overlap in these different samples. As shown in Figure 1 (a), cluster masses derived by V09 and M10 using the same proxy $M_{\text {gas }}$ show a systematic offset. The best fit is found as

$\log M_{500, \mathrm{~V} 09}=(0.99 \pm 0.06) \log M_{500, \mathrm{M} 10}-(0.07 \pm 0.04)$.

This equation can be used to convert the mass estimates of all galaxy clusters in the M10 sample to the V09 standard. Similarly, we compare the mass estimates for overlapping common galaxy clusters in V09 and P15, as shown in Figure 1 (b), and obtain the conversion equation as

$\log M_{500, \mathrm{~V} 09}=(1.06 \pm 0.03) \log M_{500, \mathrm{P} 15}+(0.02 \pm 0.02)$.

In the cluster sample of P11, there are too few clusters that overlap with V09 sample. However, there are many overlap- 
TABLE 1

RESCALED MASS AND NEWLY CALCULATED RICHNESS FOR 1191 GALAXY CLUSTERS

\begin{tabular}{rcrccccccc}
\hline ID & $\begin{array}{l}\text { R.A. } \\
(\mathrm{deg})\end{array}$ & $\begin{array}{c}\text { Decl. } \\
(\mathrm{deg})\end{array}$ & $z$ & $\begin{array}{c}M_{500} \\
\left(10^{14} M_{\odot}\right)\end{array}$ & $\begin{array}{c}\sigma_{M_{500}} \\
\left(10^{14} M_{\odot}\right)\end{array}$ & $\begin{array}{c}r_{500} \\
(\mathrm{Mpc})\end{array}$ & $\begin{array}{c}\sigma_{r_{500}} \\
(\mathrm{Mpc})\end{array}$ & $R_{L *, 500}$ & Reference \\
$(1)$ & $(2)$ & \multicolumn{1}{c}{$(3)$} & $(4)$ & $(5)$ & $(6)$ & $(7)$ & $(8)$ & \multicolumn{1}{c}{$(9)$} & $(10)$ \\
\hline 1 & 0.49367 & 12.06612 & 0.1995 & 4.40 & 0.42 & 1.08 & 0.04 & 86.20 & $\mathrm{P} 11, \mathrm{P} 15$ \\
2 & 0.79826 & -6.09169 & 0.2328 & 7.20 & 0.44 & 1.26 & 0.03 & 105.02 & $\mathrm{P} 11, \mathrm{P} 15$ \\
3 & 0.95698 & 2.06647 & 0.0967 & 2.57 & 0.26 & 0.94 & 0.03 & 60.13 & $\mathrm{P} 11, \mathrm{P} 15$ \\
4 & 1.24353 & 11.70091 & 0.0763 & 2.00 & 0.30 & 0.87 & 0.05 & 23.18 & $\mathrm{P} 11$ \\
5 & 1.34984 & 16.21926 & 0.1155 & 3.70 & 0.56 & 1.05 & 0.06 & 67.62 & $\mathrm{P} 11$ \\
6 & 1.58453 & 10.86429 & 0.1680 & 4.46 & 0.41 & 1.10 & 0.03 & 68.22 & $\mathrm{P} 11, \mathrm{P} 15$ \\
7 & 2.04332 & 2.02009 & 0.3665 & 6.44 & 0.70 & 1.16 & 0.04 & 68.70 & $\mathrm{H} 13, \mathrm{P} 15$ \\
8 & 2.33609 & 6.82309 & 0.2378 & 5.80 & 0.66 & 1.17 & 0.05 & 112.20 & $\mathrm{P} 15$ \\
9 & 2.51443 & 17.77082 & 0.1714 & 3.76 & 0.59 & 1.04 & 0.06 & 64.06 & $\mathrm{P} 15$ \\
10 & 2.57239 & 6.64203 & 0.2648 & 5.68 & 0.72 & 1.15 & 0.05 & 53.00 & $\mathrm{P} 15$ \\
\hline
\end{tabular}

Notes: Column (1): sequence number; Column (2) and (3): R.A. (J2000) and Decl. (J2000) of cluster; Column (4): spectroscopic redshift of cluster; Column (5) and (6): rescaled cluster mass $M_{500}$ and its uncertainty $\sigma_{M_{500}}$; Column (7) and (8): recalculated cluster radius $r_{500}$ and its uncertainty $\sigma_{r_{500}}$ from $M_{500}$; Column (9): newly defined cluster richness $R_{L *, 500}$; Column (10): reference for original mass estimate: V09 for Vikhlinin et al. (2009a), M10 for Mantz et al. (2010), P11 for Piffaretti et al. (2011), H13 for Hasselfield et al. (2013), P15 for Planck Collaboration et al. (2015), T14 for Takev et al. (2013, 2014).

(This table is available in its entirety in a machine-readable form in the online journal. A portion is shown here for guidance regarding the form and content.)

ping common clusters with the P15 catalog. We can rescale the cluster masses of the P11 sample to the P15 scale first, and then convert to the V09 standard. Comparing the mass estimates of the common clusters overlapping in the P11 and P15 samples shown in Figure1(c) gives

$\log M_{500, \mathrm{P} 15}=(0.90 \pm 0.02) \log M_{500, \mathrm{P} 11}+(0.15 \pm 0.01)$.

Combining the above two conversion equations, we get

$\log M_{500, \mathrm{~V} 09}=(0.95 \pm 0.03) \log M_{500, \mathrm{P} 11}+(0.18 \pm 0.02)$.

The H13 sample of the SZ clusters has even fewer overlaps with the samples of V09 and P15. However, there are some common clusters overlapping in the H13 and P11 samples. We compare their mass estimates as shown in Figure 11(d), and get

$\log M_{500, \mathrm{P} 11}=(0.98 \pm 0.30) \log M_{500, \mathrm{H} 13}-(0.19 \pm 0.30)$.

When it is combined with Equation (5), we get the conversion equation to the V09 standard as

$\log M_{500, \mathrm{~V} 09}=(0.93 \pm 0.29) \log M_{500, \mathrm{H} 13}+(0.00 \pm 0.29)$.

The same holds for the clusters in the T14 sample. The best fit between the T14 mass and the P11 mass as shown in Figure 1(e) is

$\log M_{500, \mathrm{P} 11}=(1.04 \pm 0.08) \log M_{500, \mathrm{~T} 14}-(0.01 \pm 0.02)$,

and the conversion to the V09 standard is through

$\log M_{500, \mathrm{~V} 09}=(0.99 \pm 0.08) \log M_{500, \mathrm{~T} 14}+(0.17 \pm 0.03)$.

The masses of clusters thus scaled in Figure 1 a)-(e) have excellent consistency, as shown in Figure 1 f), which shows a scatter of only 0.1 dex, i.e. $\sigma_{\log M_{500}}=0.10$. Most of the galaxy clusters from the V09, M10, P11, H13, and P15 samples have a rescaled mass of $M_{500}>2 \times 10^{14} M_{\odot}$, while clusters in the T14 sample often have a smaller mass of $0.3 \times$ $10^{14} M_{\odot}<M_{500}<2 \times 10^{14} M_{\odot}$.

The rescaled masses of the galaxy clusters from the six sam-

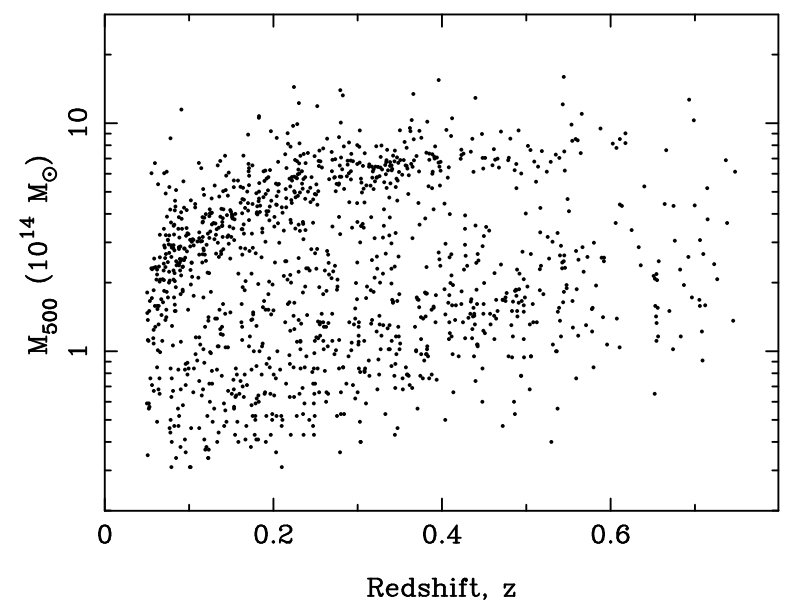

FIG. 2.- The masses for 1191 clusters in the composite calibration sample are in the range of $0.3-20 \times 10^{14} M_{\odot}$, and the redshifts are distributed up to 0.75 .

ples will be used to calibrate the optical mass proxy, which will be derived from the SDSS data. Therefore we limit the composite sample to galaxy clusters in the SDSS sky region, which must have a spectroscopic redshift either in the NASA/IPAC Extragalactic Database or from the SDSS spectroscopic observations (Alam et al. 2015). We also limit the sample to a mass of $M_{500}>0.3 \times 10^{14} M_{\odot}$ and in the redshift range of $0.05<z<0.75$. The lower redshift limit is set due to a photometry problem for very bright galaxies in the SDSS data, and the redshift upper limit is considered for the reliable detection of cluster galaxies in the SDSS data. We further visually inspect the color images ${ }^{1}$ and exclude nine clusters that are seriously contaminated by very bright exposure-saturated stars. Finally we get 29, 64, 584, 50, 412, and 370 clusters from the V09, M10, P11, H13, T14 and P15 samples, respectively. After the overlapping common clusters are merged with their masses estimated from uncertaintyweighted average, the composite calibration sample consists

\footnotetext{
${ }^{1}$ http://skyserver.sdss.org/dr12/en/tools/chart/navi.aspx
} 


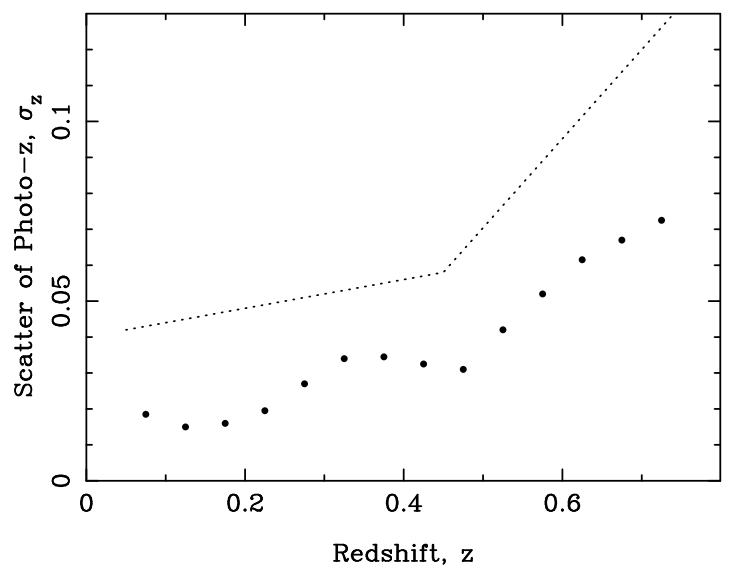

FIG. 3.- Data scatter $\sigma_{z}$ of photo- $z$ varies with redshift, obtained from the comparison of spectroscopic redshifts and estimated photo- $z \mathrm{~s}$ of a large SDSS galaxies. The dotted line represents the half thickness $\Delta z$ of the photo$z$ slice defined by Equation 10 for discrimination of member galaxies.

of 1191 clusters, as listed in Table 1 The cluster radius $r_{500}$ are re-estimated from the rescaled mass $M_{500}$ by using Equation (11). The distributions of their masses and redshifts are shown in Figure 2 .

\subsection{Optical luminosity and richness of galaxy clusters}

We discriminate the cluster member galaxies and calculate the total optical luminosity of clusters by using the SDSS spectroscopic and photometric data following our previous procedures in Wen et al. (2012, hereafter WHL12) but with a few improvements. The cluster richness is defined from the optical luminosity, which can act as the optical mass proxy for galaxy clusters.

\subsubsection{Discrimination of cluster member galaxies}

We only consider the bright member galaxies of $M_{r}^{e}(z) \leq$ -20.5 . Here, $M_{r}^{e}$ is the evolution-corrected absolute magnitude in the $r$ band, $M_{r}^{e}(z)=M_{r}(z)+Q z$. Assuming the passive evolution of a galaxy population formed at $z_{f}=2$ (Lin et al. 2006; Crawford et al. 2009) and adopting a stellar population synthesis model (Bruzual \& Charlot 2003) with the initial mass function of Chabrier (2003) and solar metallicity, we obtain the value of $Q \sim 1.16$ at redshift $z<0.8$. The bright member galaxies of a cluster can be discriminated around a brightest cluster galaxy (BCG) in a small redshift range, i.e. a redshift slice.

To discriminate the cluster galaxies, the spectroscopic redshifts are taken if they are available in the SDSS DR12 (Alam et al. 2015); otherwise, photometric redshifts (photo$z \mathrm{~s}$ hereafter) will be used. For the objects classified as galaxies with only photo- $z$ s, we remove those with deblending problems and those marked as 'SATURATED' using the flags ${ }^{2}$, and also discard those objects with a large photo- $z$ error $z \operatorname{Err}>0.08(1+z)$. These selection procedures remove most of the contamination from stars and galaxies with a bad photometry.

For galaxies with spectroscopic redshifts, only those within a redshift slice of $c \Delta z /(1+z)=2500 \mathrm{~km} \mathrm{~s}^{-1}$ are considered as member galaxies. Here $c$ is the speed of light, and $z$ is the redshift of a cluster.

2 (flags \& 0x20) $=0$ and (flags \& 0x80000) $=0$ and ((flags \& $0 \mathrm{x} 400000000000)=0$ or $\left.\operatorname{psfmagerr}_{r}<=0.20\right)$ and $(($ flags $\& 0 \mathrm{x} 40000)=0)$
For most member galaxies, their photo- $z \mathrm{~s}$ were estimated by optical magnitudes in five bands of the SDSS and have an uncertainty much larger than those of spectroscopic redshift (Csabai et al. 2007). The data scatter of photo- $z$ varies with redshift, as shown in Figure 3 , and is smaller than 0.035 at a redshift of $z<0.5$ but increases to $\sigma_{z} \sim 0.075$ at a redshift of $z>0.7$. Only galaxies within the redshift slice of $z \pm \Delta z$ are considered as member galaxies of a cluster at a redshift of $z$. In Wen et al. (2009) and Wen et al. (2012) the half thickness of the slice is taken as $\Delta z=0.04(1+z)$, and the majority of the member galaxies can be well discriminated for clusters with a redshift of $z<0.42$. However, due to the large uncertainty of photo- $z$, such a slice leads to incomplete recognizance of member galaxies for clusters at higher redshifts. Here we adopt the photo- $z$ slice in the form of

$$
\Delta z=\left\{\begin{array}{ll}
0.04(1+z) & \text { for } z \leq 0.45 \\
0.248 z-0.0536 & \text { for } z>0.45
\end{array},\right.
$$

which is about $1.75-2.00 \sigma_{z}$ and by which most cluster member galaxies can be identified up to a high redshift. Such a larger photo- $z$ slice leads to a slightly higher completeness for identification of member galaxies but introduces more contamination.

Compared to Wen et al. (2012), we improve the discrimination of member galaxies in three aspects: (a) spectroscopic redshifts are considered for member galaxies; (b) the evolution of absolute magnitudes is improved from $Q=1.62 \pm$ 0.30 (Blanton et al. 2003) to the current value of $Q=1.16$; and (c) the thickness of the photo- $z$ slice at high redshifts is enlarged according to the photo- $z$ scatter.

\subsubsection{Total $r$-band luminosity of cluster member galaxies and background estimation}

In general for each known cluster, the total $r$-band luminosity can be calculated by summing the luminosity of recognized member galaxies. The total luminosity is measured in units of $L^{*}$ which is the evolved characteristic luminosity of galaxies in the $r$-band defined by $L^{*}(z)=L^{*}(0) 10^{0.4 Q z}$, here again $Q=1.16$.

However, the contamination by background field galaxies has to be subtracted. To estimate the local background, we follow the method analogous to Popesso et al. (2004). For each cluster, we take the BCG as the center of a galaxy cluster, and then divide the annulus between 2-4 Mpc from the BCG into 48 sectors with equal areas. Within the same magnitude limit and the same photo- $z$ slice, the field galaxies in these sectors fainter than the second brightest cluster member galaxies are used to calculate the background. We get the total $r$-band luminosity of bright galaxies in each sector, and estimate the mean and deviation $\sigma$. The sectors with a luminosity obviously deviating from the mean by more than $3 \sigma$ are discarded, and the mean is recalculated for the local background which is scaled and subtracted from the total luminosity.

\subsubsection{Scaling relations between the optical data and mass}

The goal here is to establish the scaling relation between the cluster mass and the luminosity or richness derived from optical data. As shown by Wen et al. (2012), if there are merely optical survey data for a cluster, a few steps are needed to derive the richness from optical data: (1) discriminate cluster member galaxies and get their redshifts; (2) get the optical luminosity $L_{1 \mathrm{Mpc}}$ from member galaxies within $1 \mathrm{Mpc}$, and then derive the cluster radius $r_{500}$; and (3) get the optical lu- 

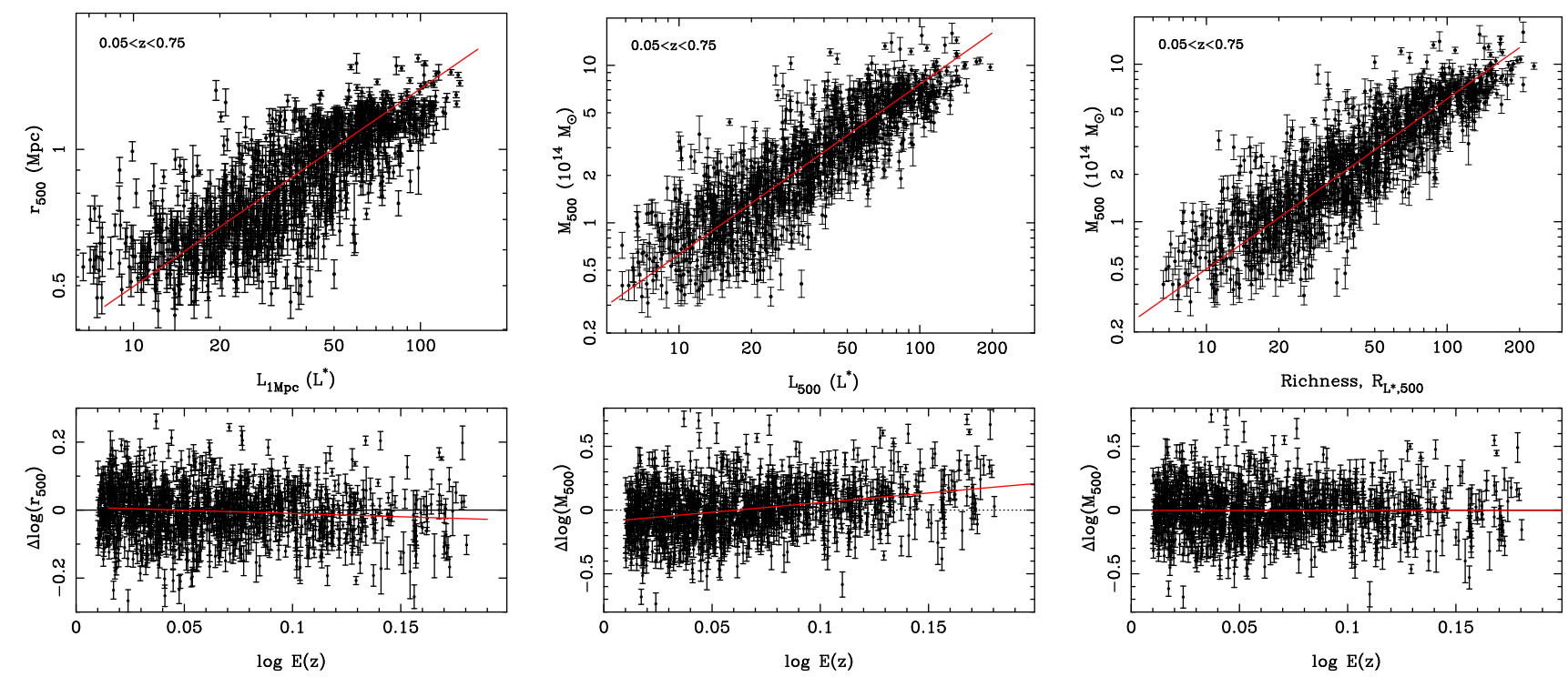

FIG. 4. - Upper panels are the scaling relations between cluster parameters derived from the optical data (the X-axis) and cluster radius or mass (the Y-axis) for the 1191 clusters from the composite calibration sample. The data scatters are plotted in the lower panels against log $E(z)$ to check their possible evolution.

minosity $L_{500}$ within $r_{500}$, and then the richness defined as $R_{L *, 500}=L_{500} / L^{*}$.

First, to estimate $r_{500}$ from optical data, we have to establish the relation between $r_{500}$ and an optical observable that is the total $r$-band luminosity $L_{1 \mathrm{Mpc}}$ (in units of $L^{*}$, with background subtracted) of member galaxies within $1 \mathrm{Mpc}$ from the cluster center. As shown in the left panels of Figure 4, we find that the values of $r_{500}$ (in Mpc) in Table 1 are well correlated with $L_{1 \mathrm{Mpc}}$. The uncertainty of $L_{1 \mathrm{Mpc}}$ comes from the incompleteness of member galaxies and residual contamination of background galaxies, which is about $10 \%$ for rich clusters (Wen et al. 2009). By taking a typical uncertainty of $15 \%$ on $L_{1 \mathrm{Mpc}}$ for all clusters, we find the best fit to the data as

$$
\log r_{500}=(0.45 \pm 0.01) \log L_{1 \mathrm{Mpc}}-(0.76 \pm 0.01) .
$$

The slope obtained here is consistent with that in Wen et al. (2012).

Some authors have shown that the scaling relation between $M_{500}$ (or $r_{500}$ ) and an optical observable (e.g., richness) may evolve with redshift (Lin et al. 2004; Andreon \& Congdon 2014; Wiesner et al. 2015), for example, due to higher concentration of the light-to-mass profiles at lower redshifts (Gu et al. 2013). On the other hand, bright member galaxies of $M_{e} \leq-20.5$ are incomplete in the SDSS photometric data at $z>0.45$, so that the total luminosity could be underestimated. The 1191 clusters in the composite calibration sample are in a very large redshift range, allowing us to clarify any redshift evolution of the scaling relations. For the scaling relation $r_{500}-L_{1 \mathrm{Mpc}}$, we calculate the deviation of $r_{500}$ from the relation: $\Delta \log r_{500}=\log r_{500}-\left(0.45 \log L_{1 \mathrm{Mpc}}-0.76\right)$ and find that $\Delta \log r_{500}$ is only slightly evolving with the redshift in the form of (see the lower left panel in Figure 4)

$$
\Delta \log r_{500}=(-0.20 \pm 0.06) \log E(z)+(0.01 \pm 0.01),
$$

where $E(z)=\sqrt{\Omega_{\Lambda}+\Omega_{m}(1+z)^{3}}$. Including this small correction, the radius $r_{500}$ is related to $L_{1 \mathrm{Mpc}}$ and $z$ by

$$
\begin{aligned}
\log r_{500} & =(0.45 \pm 0.01) \log L_{1 \mathrm{Mpc}}-(0.75 \pm 0.01) \\
& -(0.20 \pm 0.06) \log E(z)
\end{aligned}
$$

We calculate the total $r$-band luminosity $L_{500}$ (also in units of $L^{*}$, with background subtracted) of member galaxies within the radius of $r_{500}$ for the 1191 clusters by using the SDSS data. As shown in the upper middle panel of Figure 4 there exists excellent correlation between $L_{500}$ and the cluster mass $M_{500}$ (in units of $10^{14} M_{\odot}$ ), and the best fit is

$$
\log M_{500}=(1.08 \pm 0.02) \log L_{500}-(1.28 \pm 0.02) .
$$

Again, to check for the possible evolution with redshift, the deviations of $M_{500}$ from the above fitted relation, $\Delta \log M_{500}=\log M_{500}-\left(1.08 \log L_{500}-1.28\right)$, are plotted against $E(z)$ in the middle lower panel of Figure 4 , which shows a correlation in the form of

$$
\Delta \log M_{500}=(1.51 \pm 0.14) \log E(z)-(0.09 \pm 0.01),
$$

Though the total $r$-band luminosity $L_{500}$ in principle may slightly be underestimated for clusters of $z>0.45$ (i.e., $\log E(0.45)=0.104)$, the $\Delta \log M_{500}$ has to be so corrected. We then can define the richness of the galaxy cluster from the total $r$-band luminosity $L_{500}$, which can act as a well-calibrated mass proxy in the optical band, as

$$
R_{L *, 500}=L_{500} E(z)^{1.40} .
$$

Such a richness is independent of redshift and is valid in the redshift range of $0.05<z<0.75$, as shown in the right panels of Figure 4 The thus-calculated richness $R_{L *, 500}$ for galaxy clusters in the composite calibration sample is listed in Table 1. The scaling relation between the cluster mass and the richness is

$$
\log M_{500}=(1.08 \pm 0.02) \log R_{L *, 500}-(1.37 \pm 0.02),
$$

which is consistent with but more accurate than the massrichness relation obtained in our previous work (Wen et al. 2012) and the $M_{500}-\lambda$ relation given by Rykoff et al. (2012). The scatter of mass values is about 0.17 dex, i.e. $\sigma_{\log M_{500}}=$ 0.17 . Taking out the inherent scatter $\sigma_{\log M_{500}}=0.10$ of the rescaled masses of the composite calibration sample, the mass uncertainty estimated by $R_{L *, 500}$ should be $\sigma_{\log M_{500}}=0.14$.

Because clusters in the composite calibration sample are 

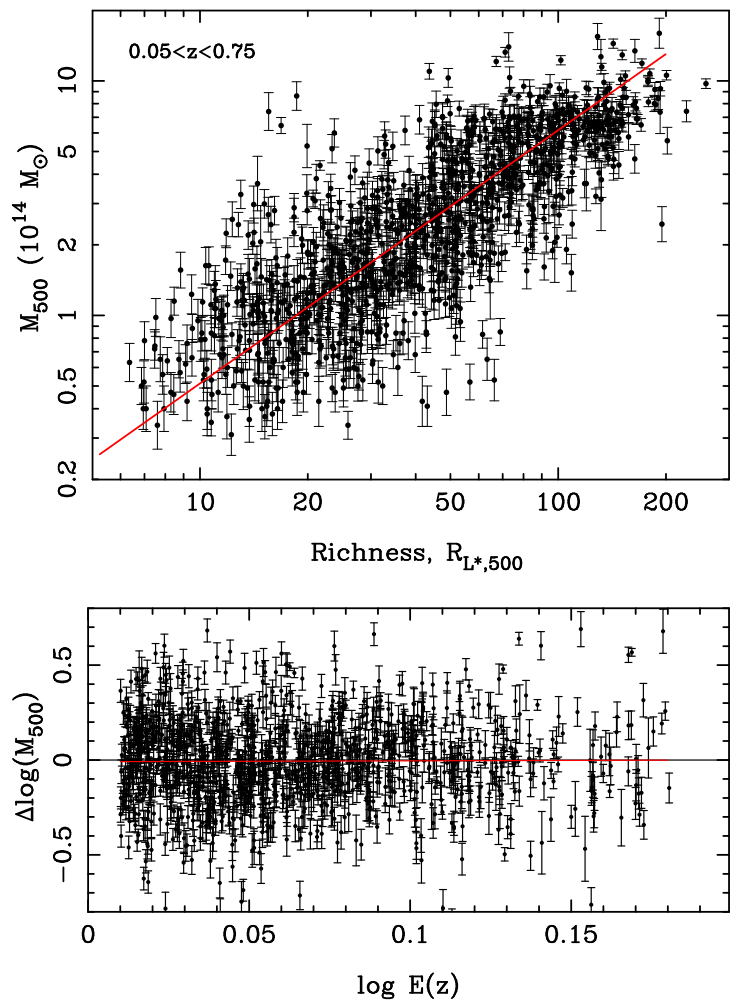

FIG. 5.- Correlation between $M_{500}$ and the directly optically estimated $R_{L *, 500}$ for the 1191 clusters in Table 1 Different from the right panel of Figure 4 the values of $r_{500}$ and $R_{L *, 500}$ are both estimated from merely optical data.

distributed in a wide range of mass and redshift, the scaling relations we derived above should be valid for clusters in the SDSS in the redshift ranges of $0.05<z<0.75$ with a mass of $M_{500}>0.3 \times 10^{14} M_{\odot}$.

\subsection{Goodness of optical mass proxies}

How good is the richness $R_{L *, 500}$ estimated from just the optical data (i.e., $r_{500}$ is unknown previously) acting as a mass proxy? To get a quantitative estimate, we estimated the $L_{1 \mathrm{Mpc}}$ of 1191 clusters in the composite calibration sample, and then get $r_{500}$ from the scaling relation in Equation (13) so that $L_{500}$ and hence $R_{L *, 500}$ can be estimated from the member galaxies within $r_{500}$. Obviously the uncertainty of estimated $r_{500}$ is propagated to $L_{500}$ and then $R_{L *, 500}$. The plot for $M_{500}$ and $R_{L *, 500}$ in Figure 5 shows a mass scatter in 0.20 dex, i.e. $\sigma_{\log M_{500}}=0.20$. Taking out the inherent scatter of $\sigma_{\log M_{500}}=0.10$ for the masses estimated from the X-ray and SZ observations, the mass scaled by $R_{L *, 500}$ should have a scatter of $\sigma_{\log M_{500}}=0.17$.

Can the richness in other cluster catalogs be used as a mass proxy? If so, how good is it? To find out the answer to these questions, we investigate the optical richness based on photometric data in the cluster catalogs of the maxBCG (Koester et al. 2007), the Gaussian Mixture Brightest Cluster Galaxy (GMBCG, Hao et al. 2010), the adaptive matched filter (AMF, Szabo et al. 2011), the WHL12 (Wen et al. 2012), the red-sequence Matched-filter Probabilistic Percolation (redMapper, Rykoff et al. 2014), the Cluster finding Algorithm based on Multi-band Identification of Redsequence galaxies (CAMIRA, Oguri 2014), and the richness based on spectroscopic data by Yang et al. (2007) and
Tempel et al. (2014).

The richness is defined as the total number of galaxies in the catalogs of maxBCG, GMBCG, and redMapper, or defined as the total luminosity of member galaxies in the catalogs of the AMF and WHL12. Yang et al. (2007) adopted the characteristic luminosities or the characteristic stellar masses as the halo mass proxy of galaxy group/clusters, and here we take the halo mass for comparison. Tempel et al. (2014) provided the dynamical mass estimates based on radial velocity dispersion of member galaxies, which we will take for comparison.

We cross-match the galaxy clusters in Table 1 with clusters in the above cluster catalogs, and get the matched clusters within a projected distance of $r_{500}$ and a redshift difference of 0.05 . If more than one cluster in a catalog matches with a cluster in Table 1, we take the one with the minimum projected distance. Note that for different cluster catalogs, we take the redshift ranges up to their redshift distribution peaks. As shown in Table 2, we get different numbers of matched clusters for different catalogs. We estimate the cluster mass by using each richness and get the scatter of the mass. For comparison, the mass scatter for the same sample of matched clusters for each catalog is also calculated by using our newly defined richness. We find that the richness defined in this paper and those in redMapper and CAMIRA are equally good to act as optical mass proxy. The improvement in this paper is that the newly defined richness has the redshift evolution corrected and is good for a wide range of redshifts up to $z \sim 0.75$.

\section{UPDATE OF THE WHL12 CLUSTER CATALOG}

We identified 132,684 galaxy clusters by using the photo$z$ of galaxies from SDSS DR8 (Wen et al. 2012). Clusters were identified if they have a richness $\geq 12$ and the number of member galaxies $N_{200} \geq 8$ within a radius of $r_{200}$. Here, $r_{200}$ is the radius within which the mean density of a cluster is 200 times of the critical density of the universe. The cluster richness $R_{L *}$ was defined to be the $r$-band total luminosity (in units of $L^{*}$ ) of member galaxies with $M_{r}^{e} \leq-20.5$ within $r_{200}$.

Two reasons lead us to update the WHL12 cluster catalog. First, the latest SDSS DR12 (Alam et al. 2015) released spectroscopic redshifts for about 2.30 million galaxies, of which about 1.35 million are luminous red galaxies (LRGs). In general LRGs are massive galaxies, and many of them are the BCGs or the bright member galaxies of clusters in the WHL12. We can update these spectroscopic redshifts for most of the WHL12 clusters. Second, the newly defined richness can act as an excellent mass proxy, and should be calculated for all clusters in the WHL12 catalog. In addition, by using the SDSS DR12 data, we can find rich galaxy clusters at high redshifts.

\subsection{Update on spectroscopic redshifts and the BCGs}

In general, we take the spectroscopic redshift of the BCG $z_{\mathrm{s}, \mathrm{BCG}}$ as the redshift of a cluster. SDSS DR12 has spectroscopic redshifts for about 2.30 million galaxies (Alam et al. 2015). Simple cross-matching the BCGs of the WHL12 clusters with the galaxies with spectroscopic data in the SDSS DR12 gives the redshifts for 84,760 (64\%) clusters.

We then try to get spectroscopic redshifts of bright member galaxies. For clusters with available $z_{\mathrm{s}, \mathrm{BCG}}$, we consider only galaxies within $r_{500}=2 / 3 r_{200}$ from the BCG and within $2500 \mathrm{~km} \mathrm{~s}^{-1}$ from $z_{\mathrm{s} \text {, BCG }}$ in the rest frame. Here $r_{200}$ can be found in Table 1 of Wen et al. (2012). For clusters without 
TABLE 2

COMPARISON OF DATA SCATTER FOR MASSES ESTIMATED FROM DIFFERENT RICHNESSES IN DIFFERENT CLUSTER CATALOGS.

\begin{tabular}{|c|c|c|c|c|}
\hline Cluster catalog & $\begin{array}{c}\text { Redshift range } \\
\text { for matching }\end{array}$ & $\begin{array}{c}\text { Number of } \\
\text { matched clusters }\end{array}$ & $\begin{array}{c}\sigma_{\log M_{500}} \\
\text { scaled from ref. }\end{array}$ & $\begin{array}{c}\sigma_{\log M_{500}} \\
\text { from this work }\end{array}$ \\
\hline maxBCG & $0.10<z<0.30$ & 325 & 0.29 & 0.20 \\
\hline GMBCG & $0.10<z<0.37$ & 502 & 0.27 & 0.19 \\
\hline $\mathrm{AMF}$ & $0.05<z<0.38$ & 402 & 0.26 & 0.20 \\
\hline WHL12 & $0.05<z<0.42$ & 799 & 0.24 & 0.20 \\
\hline redMapper & $0.08<z<0.55$ & 610 & 0.17 & 0.18 \\
\hline CAMIRA & $0.1<z<0.6$ & 647 & 0.18 & 0.19 \\
\hline Yang et al. & $z<0.2$ & 195 & 0.56 & 0.19 \\
\hline Tempel et al. & $z<0.2$ & 149 & 0.23 & 0.19 \\
\hline This paper & $0.05<z<0.75$ & 1191 & - & 0.20 \\
\hline
\end{tabular}

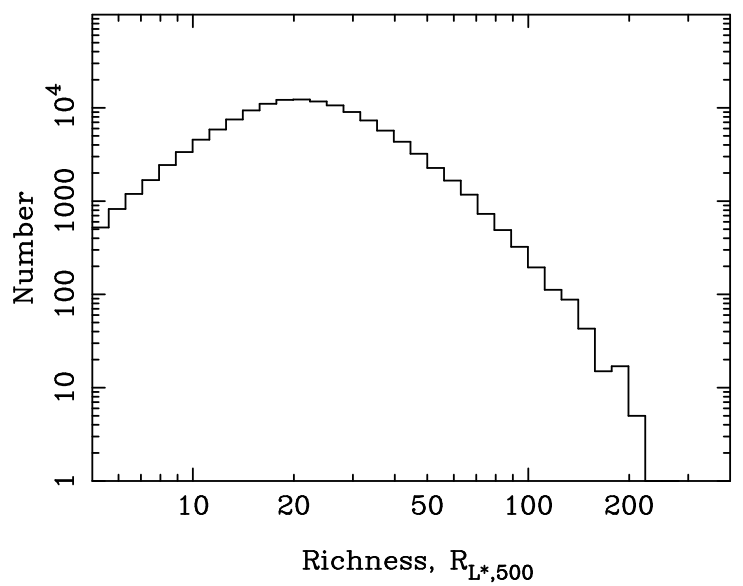

FIG. 6.- Distribution of the newly defined richness $R_{L *, 500}$ for the WHL12 clusters.

$z_{\mathrm{s}, \mathrm{BCG}}$, we consider the galaxies within $r_{500}=2 / 3 r_{200}$ from the BCGs and within a redshift difference of $0.03(1+z)$ from $z_{\mathrm{p}}$, the photo- $z$ of clusters in Wen et al. (2012). This small redshift difference is about 2.0-2.5 times the uncertainty of the cluster photo- $z$. The mean spectroscopic redshifts of the member galaxies (including the BCG) is regarded to be the spectroscopic redshift of a cluster. Through this process, we get spectroscopic redshifts for 95,684 clusters, which is about $72 \%$ in the WHL catalog. In the SDSS spectroscopic survey region, $85 \%$ of the WHL clusters have spectroscopic redshifts obtained. Clusters located at low Galactic latitudes have no spectroscopic redshifts yet.

The position and the BCG have been updated for some clusters as well. In Wen et al. (2012), all objects with the deblending problems or marked as "SATURATED" (see the flags in Section 2.2) were removed from the photometric data in the procedure for identifying galaxy clusters. The true BCGs that have such a flag or catastrophic photo- $z$ have now been included by using the spectroscopic redshifts. If a galaxy is brighter than the BCG of an original WHL12 cluster within $r_{200}$ and has a redshift difference of $0.03(1+z)$ and is surrounded by other member galaxies in the SDSS color image, we take it to be the new BCG of the cluster. We hence update the BCGs for 3128 clusters.

\subsection{Update on cluster richness}

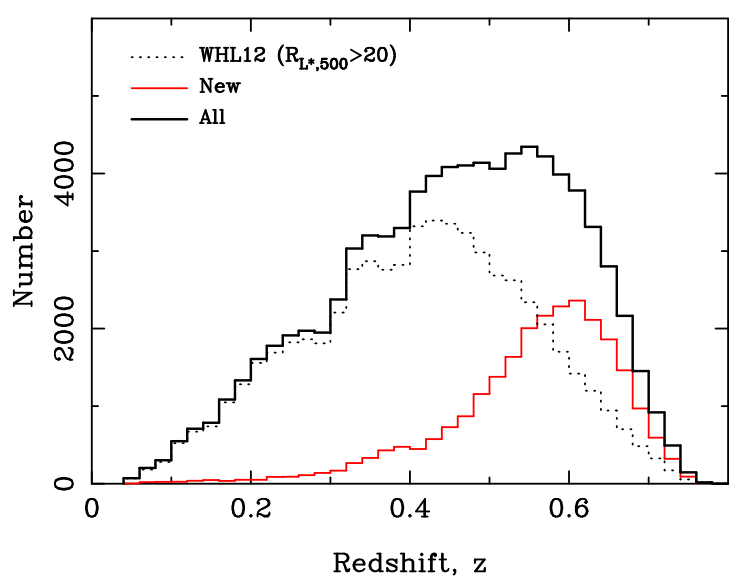

FIG. 7.- Redshift distribution of the newly identified clusters, compared with the WHL12 clusters of $R_{L *, 500} \geq 20$ in the SDSS spectroscopic survey region.

By using the scaling relations we obtained in Section 2.2.3, we update the richness of galaxy clusters in the WHL12 cata$\log$ via the following steps:

$$
L_{1 \mathrm{Mpc}} \rightarrow r_{500} \rightarrow L_{500} \rightarrow R_{L *, 500}
$$

That is to say, we have to estimate $r_{500}$ from $L_{1 \mathrm{Mpc}}$, and then get $L_{500}$ and $R_{L *, 500}$.

The updated parameters are listed in Table 3, where the cluster names, coordinates of the BCG, cluster redshift, and the $r$-band magnitude, as well as the newly estimated radius $r_{500}$ and newly defined richness $R_{L *, 500}$ are listed. The number of member galaxies $N_{500}$ within $r_{500}$ and the number of these galaxies with spectroscopic redshifts $N_{500 \text {,sp }}$ are given for reference. The distribution of $R_{L *, 500}$ is shown in Figure 6, it has a peak around $R_{L *, 500}=20$. We noticed that 5183 clusters in the WHL12 catalog has a low new richness of $R_{L *, 500}<8$.

\subsection{Complementary galaxy clusters at high redshifts}

Identification of galaxy clusters at high redshifts was incomplete due to the large uncertainty of the photometric redshift, so that the the number of clusters in the WHL12 catalog drops down at redshifts of $z>0.42$ (Wen et al. 2012, see also Figure 7). Here we identify complementary rich clusters around bright galaxies which have spectroscopic redshifts measured in the SDSS DR12, with following procedures: 
TABLE 3

THE WHL12 CLUSTER CATALOG WITH UPDATED PARAMETERS

\begin{tabular}{ccccccccccccc}
\hline Cluster name & $\begin{array}{c}\text { R.A. } \\
(\mathrm{deg})\end{array}$ & $\begin{array}{c}\text { Decl. } \\
(\mathrm{deg})\end{array}$ & $z_{p}$ & $z_{s}$ & $r_{\mathrm{BCG}}$ & $\begin{array}{c}r_{200} \\
(\mathrm{Mpc})\end{array}$ & $R_{L *}$ & $N_{200}$ & $\begin{array}{c}r_{500} \\
(\mathrm{Mpc})\end{array}$ & $R_{L *, 500}$ & $N_{500, \mathrm{sp}}$ & $N_{500}$ \\
& $(2)$ & $(3)$ & $(4)$ & $(5)$ & $(6)$ & $(7)$ & $(8)$ & $(9)$ & $(10)$ & $(11)$ & $(12)$ & $(13)$ \\
\hline WHL J000000.6+321233 & 0.00236 & 32.20925 & 0.1274 & -1.0000 & 14.92 & 1.72 & 70.63 & 24 & 1.01 & 68.28 & 0 & 19 \\
WHL J000002.3+051718 & 0.00957 & 5.28827 & 0.1696 & 0.1694 & 16.20 & 0.94 & 17.48 & 9 & 0.72 & 24.46 & 1 & 9 \\
WHL J000003.1-033245* & 0.01276 & -3.54578 & 0.5968 & 0.5973 & 20.14 & 1.00 & 19.19 & 11 & 0.83 & 49.33 & 2 & 13 \\
WHL J000003.3+311354 & 0.01377 & 31.23175 & 0.5428 & 0.5293 & 20.17 & 0.87 & 14.27 & 8 & 0.63 & 25.43 & 2 & 9 \\
WHL J000003.5+314708 & 0.01475 & 31.78564 & 0.0932 & 0.0916 & 15.18 & 0.94 & 16.97 & 9 & 0.69 & 21.14 & 1 & 11 \\
WHL J000004.7+022826 & 0.01945 & 2.47386 & 0.4179 & -1.0000 & 19.32 & 0.95 & 13.71 & 10 & 0.54 & 6.60 & 0 & 3 \\
WHL J000005.5+354610 & 0.02303 & 35.76957 & 0.4762 & -1.0000 & 19.59 & 0.91 & 15.58 & 9 & 0.63 & 19.13 & 0 & 6 \\
WHL J000006.0+152548 & 0.02482 & 15.42990 & 0.1656 & 0.1731 & 16.60 & 1.13 & 23.53 & 19 & 0.79 & 19.53 & 2 & 12 \\
WHL J000006.3+221220 & 0.02643 & 22.20558 & 0.3985 & -1.0000 & 19.36 & 0.84 & 12.73 & 11 & 0.52 & 11.92 & 0 & 7 \\
WHL J000006.6+100648 & 0.02755 & 10.11333 & 0.3676 & 0.3747 & 19.07 & 0.93 & 16.73 & 13 & 0.70 & 23.52 & 2 & 12 \\
\hline
\end{tabular}

Note. Column (1): Cluster name, the name with '*' means the BCG is updated; Column (2) and (3): R.A. (J2000) and Decl. (J2000) of cluster; Column (4): photometric redshift of cluster; Column (5): spectroscopic redshift of cluster, -1.0000 means not available; Column (6): $r$-band magnitude of the BCG; Column (7): original $r_{200}$ of cluster (Mpc); Column (8): original richness; Column (9): original number of member galaxy within $r_{200}$; Column (10): cluster radius $r_{500}$ derived from optical luminosity; Column (11): newly defined cluster richness, $R_{L *, 500}$; Column (12): number of member galaxies with spectroscopic redshifts within $r_{500}$; Column (13): number of all member galaxies within $r_{500}$. Column (4) and (7)-(9) are from Wen et al. (2012), Column (10)-(13) are derived in this paper.

(This table is available in its entirety in a machine-readable form in the online journal. A portion is shown here for guidance regarding the form and content.)

TABLE 4

NEWLY IDENTIFIED RICH CLUSTERS AT HIGH REDSHIFTS

\begin{tabular}{ccccccccr}
\hline Cluster name & $\begin{array}{c}\text { R.A. } \\
(\mathrm{deg})\end{array}$ & $\begin{array}{c}\text { Decl. } \\
(\mathrm{deg})\end{array}$ & $z$ & $r_{\mathrm{BCG}}$ & $\begin{array}{c}r_{500} \\
(\mathrm{Mpc})\end{array}$ & $R_{L *, 500}$ & $N_{500, \mathrm{sp}}$ & $N_{500}$ \\
\hline WH J000004.2+0219411 & 0.01743 & 2.32800 & 0.6443 & 20.15 & 0.69 & 27.88 & 1 & 6 \\
WH J000004.3-091120 & 0.01809 & -9.18902 & 0.6028 & 19.95 & 0.70 & 34.93 & 1 & 7 \\
WH J000005.9+100144 & 0.02452 & 10.02896 & 0.6712 & 20.46 & 0.65 & 27.84 & 1 & 6 \\
WH J000006.8+195244 & 0.02824 & 19.87897 & 0.4771 & 19.88 & 0.62 & 22.75 & 2 & 7 \\
WH J000007.7+234443 & 0.03208 & 23.74541 & 0.5285 & 19.84 & 0.65 & 27.25 & 2 & 7 \\
WH J000008.2+263228 & 0.03423 & 26.54098 & 0.5462 & 20.02 & 0.64 & 26.35 & 1 & 8 \\
WH J000008.6+053257 & 0.03568 & 5.54922 & 0.5742 & 20.05 & 0.73 & 34.66 & 3 & 10 \\
WH J000009.9-072520 & 0.04132 & -7.42227 & 0.5847 & 20.78 & 0.62 & 24.19 & 1 & 8 \\
WH J000010.4+041038 & 0.04318 & 4.17714 & 0.6954 & 21.29 & 0.58 & 24.96 & 1 & 6 \\
WH J000013.5-022138 & 0.05613 & -2.36069 & 0.4959 & 19.94 & 0.61 & 22.55 & 2 & 7 \\
\hline
\end{tabular}

Note. Column (1): cluster name; Column (2) and (3): R.A. (J2000) and Decl. (J2000) of cluster; Column (4): spectroscopic redshift of cluster; Column (5): $r$-band magnitude of the BCG; Column (6): cluster radius $r_{500}$ derived from optical luminosity $L_{1 \mathrm{Mpc}}$; Column (7): cluster richness, $R_{L *, 500}$; Column (8): number of member galaxies with spectroscopic redshifts within $r_{500}$; Column (9): number of all member galaxies within $r_{500}$.

(This table is available in its entirety in a machine-readable form in the online journal. A portion is shown here for guidance regarding the form and content.)

1. Get the spectroscopic redshift of a cluster candidate. Each bright galaxy of $M_{e} \leq-20.5$ with a spectroscopic redshift is assumed to be a member galaxy of a cluster candidate. All galaxies within $0.5 \mathrm{Mpc}$ from a given galaxy and with a velocity difference in the rest frame less than $2500 \mathrm{~km} \mathrm{~s}^{-1}$ are taken to be the member galaxies of the cluster candidate. Then the redshift of the cluster is defined as the median value of the spectroscopic redshifts of these galaxies.

2. Determine the BCG. Generally, the true BCG, because it is the brightest, should have been observed in the spectroscopic survey already. However, the BCGs in some clusters may be not observed spectroscopically because of their blue colors (Crawford et al. 1999; Pipino et al. 2011) or the fiber collision limitation in the SDSS spectroscopic survey. The BCG candidates are the galaxies within $0.5 \mathrm{Mpc}$ and with a velocity difference less than $2500 \mathrm{~km} \mathrm{~s}^{-1}$ from the target galaxy or with a photo- $z$ difference of $0.03(1+z)$. The small slice of photo- $z$ is used here for the BCG selection to reduce contamination from field galaxies. The BCG of a cluster can- didate is recognized as the brightest galaxy of these galaxies.

3. Calculate the richness and judge a rich cluster. After we have the redshift of a cluster candidate and also the recognized BCG, we then find the bright member galaxies in the redshift slice defined by Equation 10, as described in Section 2.2.3 and then calculate $L_{1 \mathrm{Mpc}}$ to estimate $r_{500}$, and then get $L_{500}$ and finally $R_{L *, 500}$. We only identify rich clusters with a richness of $R_{L *, 500} \geq 20$ (beyond the peak in Figure 6) and the number of bright galaxies within $r_{500}, N_{500} \geq 6$.

4. Clean the entries. It is possible that one rich cluster can be recognized two or more times in the above procedures when targeting different bright galaxies, and hence some entries should be merged to one cluster if their redshift difference is less than 0.1 and a projected distance is less than $1.5 r_{500}$.

We find 79,498 clusters through the above procedures, of which 54,079 can match the clusters in the WHL12 catalog with a redshift difference of 0.1 and a projected distance of $1.5 r_{500}$. The remaining 25,419 are newly identified clusters, 


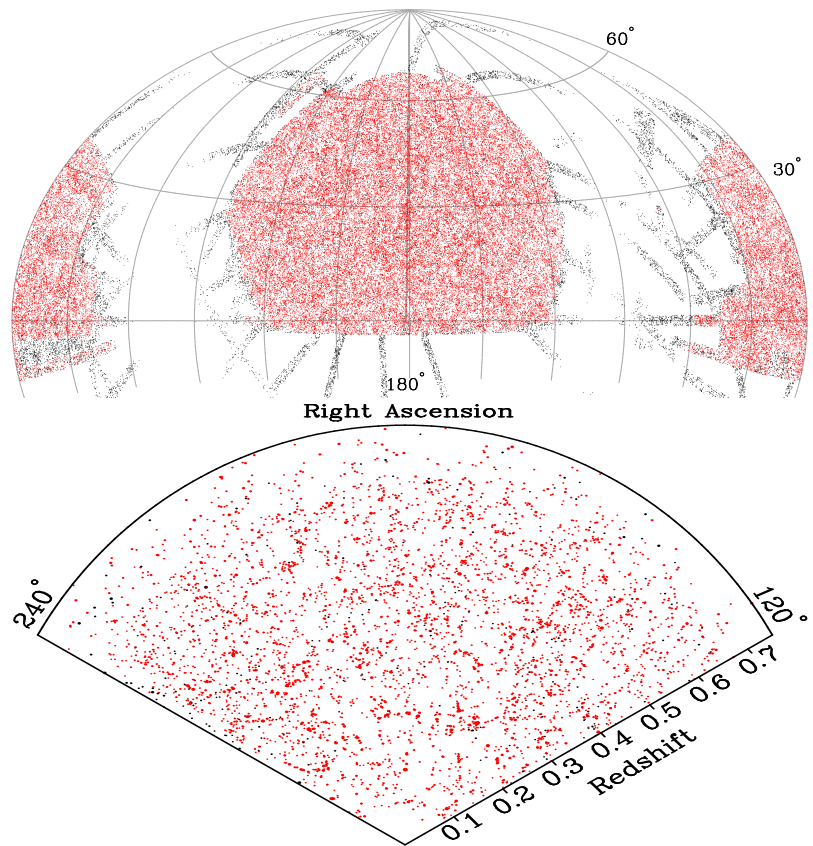

FIG. 8.- Sky coverage of rich clusters of $R_{L *, 500} \geq 20$ (upper panel) and their radial distribution in a slice $0^{\circ} \leq$ Decl. $\leq 5^{\circ}$ (lower panel). The red points are clusters with spectroscopic redshifts. The dot size in the lower panel is scaled by $\sqrt{R_{L *, 500}}$.

as listed in Table 4 Most of them have a high redshift of $z>0.4$ as shown in Figure 7

If we combine the cluster sample in the updated WHL12 catalog and the complementary catalog of newly identified 25,419 clusters, we get 158,103 clusters, of which 96,257 have a richness of $R_{L *, 500} \geq 20$. Figure 8 shows the sky coverage and radial distribution in a sky slice for clusters in the combined catalog. In the SDSS spectroscopic survey region, $89 \%$ of clusters have spectroscopic redshifts.

\section{CONCLUSIONS}

In this paper, we calibrated the optical mass proxy of galaxy clusters and applied it to update the WHL12 cluster catalog.

We collected clusters with known masses $\left(M_{500}\right)$ derived from X-ray or SZ measurements from the literature, and rescaled them for consistency. The composite mass calibration sample consists of 1191 clusters in the redshift range $0.05<z<0.75$ with mass $M_{500}>0.3 \times 10^{14} M_{\odot}$, and all of them are limited to the SDSS sky coverage to obtain the optical spectroscopic and photometric data.

We established the scaling relations between the total $r$ band optical luminosity of member galaxies $L_{1 \mathrm{Mpc}}$ within $1 \mathrm{Mpc}$ and the cluster radius $\left(r_{500}\right)$. After correcting for weak redshift dependence, the scaling relation can be used to estimate the cluster radius from optical data. The cluster mass $M_{500}$ is found to be well-correlated with the total $r$ band luminosity $L_{500}$, which evolves significantly with redshift. Considering the redshift evolution, we defined the new cluster richness as $R_{L *, 500}=L_{500} E(z)^{1.40}$, which can be used as a mass proxy independent of redshift and is valid in the redshift range of $0.05<z<0.75$. The scatter of mass estimates is about 0.17 dex.

We updated the spectroscopic redshifts for the WHL12 clusters using the SDSS DR12 spectroscopic data, and then obtain their newly defined richness. We identify 25,419 complementary clusters at high redshifts around bright galaxies with a spectroscopic redshift in the SDSS DR12. The finial combined catalog contains 158,103 clusters in total, and about $89 \%$ of them have spectroscopic redshifts in the SDSS spectroscopic survey region.

We thank the referee for helpful comments. The authors are supported by the National Natural Science Foundation (NNSF) of China (11103032 and 11473034) and the Young Researcher Grant of National Astronomical Observatories, Chinese Academy of Sciences. Funding for SDSSIII has been provided by theAlfred P. Sloan Foundation, the Participating Institutions, the National Science Foundation, and theUSDepartment of Energy. The SDSS-III Web site is http://www.sdss3.org/ SDSS-III is managed by the Astrophysical Research Consortium for the Participating Institutions of the SDSS-III Collaboration including the University of Arizona, the Brazilian Participation Group, Brookhaven National Laboratory, University of Cambridge, University of Florida, the French Participation Group, the German Participation Group, the Instituto de Astrofisica de Canarias, the Michigan State/Notre Dame/JINA Participation Group, Johns Hopkins University, Lawrence Berkeley National Laboratory, Max Planck Institute for Astrophysics, NewMexico State University, New York University, Ohio StateUniversity, Pennsylvania State University,University of Portsmouth, Princeton University, the Spanish Participation Group, University of Tokyo, University of Utah,Vanderbilt University, University of Virginia, University of Washington, and Yale University.

\section{REFERENCES}

Alam, S., Albareti, F. D., Allende Prieto, C., et al. 2015, arXiv:1501.00963

Allen, S. W., Evrard, A. E., \& Mantz, A. B. 2011, ARA\&A, 49, 409

Andreon, S., \& Congdon, P. 2014, A\&A, 568, A23

Arnaud, M., Pratt, G. W., Piffaretti, R., et al. 2010, A\&A, 517, A92

Bardeau, S., Soucail, G., Kneib, J.-P., et al. 2007, A\&A, 470, 449

Berlind, A. A., Frieman, J., Weinberg, D. H., et al. 2006, ApJS, 167, 1

Bird, C. M. 1995, ApJ, 445, L81

Blanton, M. R., Hogg, D. W., Bahcall, N. A., et al. 2003, ApJ, 592, 819

Bode, P., Ostriker, J. P., Cen, R., \& Trac, H. 2012, arXiv:1204.1762

Böhringer, H., Voges, W., Huchra, J. P., et al. 2000, ApJS, 129, 435

Böhringer, H., Schuecker, P., Guzzo, L., et al. 2004, A\&A, 425, 367

Brunetti, G., Cassano, R., Dolag, K., \& Setti, G. 2009, A\&A, 507, 661

Bruzual, G., \& Charlot, S. 2003, MNRAS, 344, 1000

Burenin, R. A., Vikhlinin, A., Hornstrup, A., et al. 2007, ApJS, 172, 561

Carlstrom, J. E., Holder, G. P., \& Reese, E. D. 2002, ARA\&A, 40, 643

Chabrier, G. 2003, PASP, 115, 763

Crawford, C. S., Allen, S. W., Ebeling, H., Edge, A. C., \& Fabian, A. C. 1999, MNRAS, 306, 857
Crawford, S. M., Bershady, M. A., \& Hoessel, J. G. 2009, ApJ, 690, 1158

Cruddace, R., Voges, W., Böhringer, H., et al. 2002, ApJS, 140, 239

Csabai, I., Dobos, L., Trencséni, M., et al. 2007, Astronomische Nachrichten, 328,852

Dahle, H. 2006, ApJ, 653, 954

Dawson, K. S., Schlegel, D. J., Ahn, C. P., et al. 2013, AJ, 145, 10

Ebeling, H., Edge, A. C., Bohringer, H., et al. 1998, MNRAS, 301, 881

Ebeling, H., Edge, A. C., \& Henry, J. P. 2001, ApJ, 553, 668

Ebeling, H., Mullis, C. R., \& Tully, R. B. 2002, ApJ, 580, 774

Eisenstein, D. J., Annis, J., Gunn, J. E., et al. 2001, AJ, 122, 2267

Finoguenov, A., Reiprich, T. H., \& Böhringer, H. 2001, A\&A, 368, 749

Gioia, I. M., Maccacaro, T., Schild, R. E., et al. 1990, ApJS, 72, 567

Girardi, M., Giuricin, G., Mardirossian, F., Mezzetti, M., \& Boschin, W. 1998, ApJ, 505, 74

Gladders, M. D., \& Yee, H. K. C. 2000, AJ, 120, 2148

Gu, L., Gandhi, P., Inada, N., et al. 2013, ApJ, 767, 157

Hao, J., McKay, T. A., Koester, B. P., et al. 2010, ApJS, 191, 254

Hasselfield, M., Hilton, M., Marriage, T. A., et al. 2013, JCAP, 7, 8 
Henry, J. P., Mullis, C. R., Voges, W., et al. 2006, ApJS, 162, 304

Horner, D. J., Perlman, E. S., Ebeling, H., et al. 2008, ApJS, 176, 374

Koester, B. P., McKay, T. A., Annis, J., et al. 2007, ApJ, 660, 239

Kravtsov, A. V., Vikhlinin, A., \& Nagai, D. 2006, ApJ, 650, 128

Lin, Y.-T., Mohr, J. J., Gonzalez, A. H., \& Stanford, S. A. 2006, ApJ, 650, L99

Lin, Y.-T., Mohr, J. J., \& Stanford, S. A. 2004, ApJ, 610, 745

Lupton, R., Gunn, J. E., Ivezić, Z., Knapp, G. R., \& Kent, S. 2001 in Astronomical Society of the Pacific Conference Series, Vol. 238, Astronomical Data Analysis Software and Systems X, ed. F. R. Harnden, Jr., F. A. Primini, \& H. E. Payne, 269

Mantz, A., Allen, S. W., Ebeling, H., Rapetti, D., \& Drlica-Wagner, A. 2010, MNRAS, 406, 1773

Merchán, M. E., \& Zandivarez, A. 2005, ApJ, 630, 759

Mullis, C. R., McNamara, B. R., Quintana, H., et al. 2003, ApJ, 594, 154

Oguri, M. 2014, MNRAS, 444, 147

Okabe, N., Takada, M., Umetsu, K., Futamase, T., \& Smith, G. P. 2010, PASJ, 62,811

Piffaretti, R., Arnaud, M., Pratt, G. W., Pointecouteau, E., \& Melin, J.-B. 2011, A\&A, 534, A109

Pipino, A., Szabo, T., Pierpaoli, E., MacKenzie, S. M., \& Dong, F. 2011, MNRAS, 417, 2817

Planck Collaboration, Ade, P. A. R., Aghanim, N., et al. 2014, A\&A, 571, A20

-. 2015, arXiv:1502.01598

Popesso, P., Biviano, A., Böhringer, H., \& Romaniello, M. 2007, A\&A, 464, 451

Popesso, P., Biviano, A., Böhringer, H., Romaniello, M., \& Voges, W. 2005, A\&A, 433, 431

Popesso, P., Böhringer, H., Brinkmann, J., Voges, W., \& York, D. G. 2004, A\&A, 423, 449

Pratt, G. W., Croston, J. H., Arnaud, M., \& Böhringer, H. 2009, A\&A, 498, 361
Reichardt, C. L., Stalder, B., Bleem, L. E., et al. 2013, ApJ, 763, 127 Reiprich, T. H., \& Böhringer, H. 2002, ApJ, 567, 716

Romer, A. K., Nichol, R. C., Holden, B. P., et al. 2000, ApJS, 126, 209

Rozo, E., Rykoff, E. S., Koester, B. P., et al. 2009, ApJ, 703, 601

Rykoff, E. S., Koester, B. P., Rozo, E., et al. 2012, ApJ, 746, 178

Rykoff, E. S., Rozo, E., Busha, M. T., et al. 2014, ApJ, 785, 104

Sifón, C., Menanteau, F., Hasselfield, M., et al. 2013, ApJ, 772, 25

Stoughton, C., Lupton, R. H., Bernardi, M., et al. 2002, AJ, 123, 485

Strauss, M. A., Weinberg, D. H., Lupton, R. H., et al. 2002, AJ, 124, 1810

Szabo, T., Pierpaoli, E., Dong, F., Pipino, A., \& Gunn, J. 2011, ApJ, 736, 21

Takey, A., Schwope, A., \& Lamer, G. 2013, A\&A, 558, A75

-. 2014, A\&A, 564, A54

Tempel, E., Tamm, A., Gramann, M., et al. 2014, A\&A, 566, A1

Trac, H., Bode, P., \& Ostriker, J. P. 2011, ApJ, 727, 94

Vikhlinin, A., Kravtsov, A., Forman, W., et al. 2006, ApJ, 640, 691

Vikhlinin, A., Burenin, R. A., Ebeling, H., et al. 2009a, ApJ, 692, 1033

Vikhlinin, A., Kravtsov, A. V., Burenin, R. A., et al. 2009b, ApJ, 692, 1060 Voit, G. M. 2005, Reviews of Modern Physics, 77, 207

von der Linden, A., Mantz, A., Allen, S. W., et al. 2014a, MNRAS, 443, 1973 von der Linden, A., Allen, M. T., Applegate, D. E., et al. 2014b, MNRAS, 439,2

Wen, Z. L., Han, J. L., \& Liu, F. S. 2009, ApJS, 183, 197

-. 2010, MNRAS, 407, 533

-. 2012, ApJS, 199, 34

Wetzel, A. R., Tinker, J. L., \& Conroy, C. 2012, MNRAS, 424, 232

Wiesner, M. P., Lin, H., \& Soares-Santos, M. 2015, arXiv:1501.06893

Yang, X., Mo, H. J., van den Bosch, F. C., et al. 2007, ApJ, 671, 153

York, D. G., Adelman, J., Anderson, Jr., J. E., et al. 2000, AJ, 120, 1579

Zhang, Y.-Y., Finoguenov, A., Böhringer, H., et al. 2008, A\&A, 482, 451

Zhao, H.-H., Jia, S.-M., Chen, Y., et al. 2013, ApJ, 778, 124 